\title{
On Commutativity of Rings Under Certain Polynomial Constraints
}

\author{
Mohammad Shadab Khan \\ Department of Commerce, Aligarh Muslim University, Aligarh, India
}

Email address:

shadabkhan33@gmail.com

\section{To cite this article:}

Mohammad Shadab Khan. On Commutativity of Rings Under Certain Polynomial Constraints. International Journal of Data Science and Analysis. Vol. 4, No. 1, 2018, pp. 13-19. doi: 10.11648/j.ijdsa.20180401.13

Received: January 9, 2018; Accepted: February 27, 2018; Published: March 19, 2018

\begin{abstract}
The pioneer theorem of Weddernburn on commutativity of division rings was proved in the very beginning of twentieth century. Aside from its own intrinsic beauty and important role in many diverse parts of algebra specially, the theorem serves as the starting point for investigations of certain kinds of conditions that render a ring commutative. A large part of the results in this area was developed in the hands of many distinguished mathematicians like Jacobson, Herstein, Kaplansky, Faith, Martindale, Nakayama, Bell and many others. The purpose of the present paper is to investigate commutativity of a ring with unity 1 satisfying certain polynomial constraints. The main result of the first section asserts that a ring is commutative if at least one of the integral exponent used in the polynomial constraints of the theorem is zero and the ring also satisfies the property $Q(n)$ Further, in the second section, commutativity of a ring with unity 1 has also been established under a set of different polynomial identities applying the most frequently used technique known as Streb's classification. Finally, in the last section, these results of the foregoing sections are further extended to a special class of rings called as one sided $s$ - unital rings.
\end{abstract}

Keywords: Associative Ring, Factor Subring, Polynomial Constraints, Nilpotent Elements, Commutators, Center of Ring, $s$ - Unital Ring and Commutativity

\section{Introduction}

Throughout the present paper, $R$ will denote an associative ring (may be without unity 1 ), $C(R)$ the commutator ideal, $P(R)$ the center and $N(R)$ the set of nilpotent elements of a ring $R$ respectively. As usual, $Z[X]$ is the totality of polynomials in $X$ with coefficients in $Z$, the ring of integers. For any $x, y \in R$, the symbol $[x, y]$ stands for commutator $x y-y x$. A ring $R$ is said to have the property $Q(n)$ if for any positive integer $n$ and for all $x, y \in R, n[x, y]=0 \Rightarrow$ $[x, y]=0$.

The present paper contains 4 sections. Section 1 deals with introduction, section 2 includes commutativity of ring with unity satisfying some identities, section 3 incorporates commutativity of ring with unity through Streb's classification and satisfying some other related identities and finally the results of the foregoing sections are extended for $s$ - unital rings in section 4 .
The famous theorem due to Jacobson [17] asserts that if every element $x$ of a ring $R$ satisfies the condition $x^{n(x)}=x$, where $n(x)>1$ is a positive integer, then $R$ is commutative. This result at the same time generalizes that every finite division ring is commutative and also the result that every Boolean ring is commutative. Among the interesting generalizations of Jacobson's theorem, Herstein [13] proved that rings satisfying the polynomial identity $(x+y)^{n}=x^{n}+y^{n}$ for some $n>1$ must have nil commutator ideal. Among other classes of rings in which ideal is known to be nil is the class of rings satisfying the polynomial identity $\left[x^{n}, y\right]=\left[x, y^{n}\right]$ for some $n>1$. It can be easily observe that this class includes the rings satisfying the identity $(x+y)^{n}=x^{n}+y^{n}$. Bell [8] proved the commutativity of a ring $R$ with unity 1 satisfying the polynomial identity $\left[x^{n}, y\right]=\left[x, y^{n}\right]$ if the additive group $(R,+)$ is $\mathrm{n}$-torsion free. Motivated by the above observations, the main purpose of this paper is to investigate 
commutativity of rings under the following polynomial constraints:

For all $y \in R$ there exist polynomials $p(X), q(X) \in Z[X] \quad$ such that $x^{r}\left[x^{n}, y\right] x^{s}=p(y)\left[x, y^{m}\right]^{k} q(y)$ for all $x \in R$ where $n>1, \quad k>0, m \geq 0, r \geq 0, s \geq 0$ are fixed non-negative integers.

A number of authors have studied commutativity of rings satisfying various special cases of the above property. The objective of the present section is to generalize these results for rings with unity 1 satisfying the above property. Further, the results are extended for one sided s- unital rings in the subsequent section.

\section{Commutativity of Ring with Unity}

Main result of this section states as follows:

Theorem 2.1 Let $R$ be a ring with unity 1 satisfying the property $x^{r}\left[x^{n}, y\right] x^{s}=p(y)\left[x, y^{m}\right]^{k} q(y)$. Further, if $R$ also satisfies the property $Q(n)$, then $R$ is commutative.

The discussion begins with the following lemmas which are pertinent for the development of the proof of the above theorem.

Lemma $2.1[18]$ If $x, y \in R$ and $[x,[x, y]]=0$, then $\left[x^{m}, y\right]=m x^{m-1}[x, y]$ for all positive integers $m$.

Lemma 2.2 [9] Let $R$ be a ring with unity 1 and let $f: R \rightarrow R$ be a polynomial function of two variables with the property $f(x+1, y)=f(x, y)$ for all $x, y \in R$. If for all $x, y \in R, x^{k} f(x, y)=0$ or $f(x, y) x^{k}=0$ for a fixed positive integer $k$, then necessarily $f(x, y)=0$.

Lemma 2.3 [19, Theorem] Let $f$ be a polynomial identity in $n$ non-commuting indeterminates $x_{1}, x_{2}, x_{3, \ldots \ldots \ldots . . .}, x_{n}$, with relatively prime integer coefficients. Then the following are equivalent

i. For ring satisfying the polynomial identity $f=0, C(R)$ is nil ideal.

ii. For every prime $p,(G F(p))_{2}$ fails to satisfy $f=0$.

iii. Every semi-prime ring satisfying $f=0$ is commutative.

Lemma 2.4 [10, Theorem] Let $R$ be a ring (may be without unity 1) and suppose for each $x, y \in R$ there exists a polynomial $f(X) \in Z[X]$ such that $[x, y]=[x, y] f(x)$. Then $R$ is commutative.

The proofs of the following lemmas are essential before developing the proof of Theorem 2.1.

Lemma 2.5 Let $R$ be a ring satisfying the property $x^{r}\left[x^{n}, y\right] x^{s}=p(y)\left[x, y^{m}\right]^{k} q(y)$, then $C(R) \subseteq N(R)$.

Proof. Suppose $R$ is a ring satisfying the given property $x^{r}\left[x^{n}, y\right] x^{s}=p(y)\left[x, y^{m}\right]^{k} q(y)$. If $r=0$, then we have

$\left[x^{n}, y\right] x^{s}=p(y)\left[x, y^{m}\right]^{k} q(y)$

Replacing $x$ by $x+1$ in the given property, we get $\left[(x+y)^{n}, y\right](x+y)^{s}=\left[x^{n}, y\right] x^{s}$.
This is a polynomial identity and it can be easily observe that $x=-e_{11}+e_{21}, y=e_{11}$ fail to satisfy this equality in $(G F(p))_{2}$, where $p$ is a prime. Hence, by Lemma 2.3 $C(R) \subseteq N(R)$. On the other hand if $s=0$, then choose $x=-e_{11}+e_{12}, y=e_{11}$ to get the required result.

Lemma 2.6 Let $R$ be a ring with unity 1 satisfying the property $x^{r}\left[x^{n}, y\right] x^{s}=p(y)\left[x, y^{m}\right]^{k} q(y)$. Moreover, $R$ satisfies the property $Q(n)$, then $N(R) \subseteq P(R)$.

Proof. Let $u \in N(R)$, then there exists a positive integer $t$ such that

$$
u^{t} \in P(R)
$$

If $t=1$, the result is obvious. Therefore, onward assume that $t>1$. Replacing $x$ by $u^{t-1}$ in the given property to get

$$
u^{r(t-1)}\left[u^{n(t-1)}, y\right] u^{s(t-1)}=p(y)\left[u^{t-1}, y^{m}\right]^{k} q(y) .
$$

Now application of (1) and the fact that $n(t-1) \geq t$, yields that

$$
p(y)\left[u^{t-1}, y^{m}\right]^{k} q(y)=0
$$

Replacement $x$ by $1+u^{t-1}$ in our hypothesis yields $\left(1+u^{t-1}\right)^{r}\left[\left(1+u^{t-1}\right)^{n}, y\right]\left(1+u^{t-1}\right)^{s}=p(y)\left[u^{t-1}, y^{m}\right]^{k} q(y)=0$. Thus, application of (2) gives

$$
\left(1+u^{t-1}\right)^{r}\left[\left(1+u^{t-1}\right)^{n}, y\right]\left(1+u^{t-1}\right)^{s}=0 . \quad \text { But since }
$$
$\left(1+u^{t-1}\right)$ is invertible, the last equation implies that

$$
\left[\left(1+u^{t-1}\right)^{n}, y\right]=0 \text { for all } y \in R
$$

Combining (1) and (3), we get

$$
0=\left[\left(1+u^{t-1}\right)^{n}, y\right]=\left[1+n u^{t-1}, y\right]
$$

i.e. $n\left[u^{t-1}, y\right]=0$, for all $y \in R$. Hence, application of property $Q(n)$ yields that $u^{t-1} \in P(R)$. This contradicts the minimality of $t$ in (1) and thus $t=1$ and $u \in P(R)$.

Proof of the Theorem. Combination of Lemma 2.5 and Lemma 2.6 gives

$$
C(R) \subseteq N(R) \subseteq P(R)
$$

Replacing $x$ by $1+x$ in the given property of the theorem to get

$$
\begin{gathered}
(1+x)^{r}\left[(1+x)^{n}, y\right](1+x)^{s}=p(y)\left[1+x, y^{m}\right]^{k} q(y) \\
=p(y)\left[x, y^{m}\right]^{k} q(y) \\
=x^{r}\left[x^{n}, y\right] x^{s} .
\end{gathered}
$$

Now application of Lemma 2.1 and (4) yields that 


$$
n[x, y](1+x)^{r+s+n-1}=n[x, y] x^{r+s+n-1}
$$

This implies that $n\left[x, y\left\{(1+x)^{r+s+n-1}-x^{r+s+n-1}\right\}\right]=0$. Application of property $Q(n)$ yields

$$
\begin{aligned}
0 & =\left[x, y\left\{(1+x)^{r+s+n-1}-x^{r+s+n-1}\right\}\right] \\
& =[x, y]\left\{(1+x)^{r+s+n-1}-x^{r+s+n-1}\right\}
\end{aligned}
$$

This is a polynomial identity which can be written in the form of $[x, y]=[x, y] f(x)$ for some $f(X) \in X Z[X]$. Hence, $R$ is commutative by Lemma 2.4.

The following corollary is an immediate consequence of the above theorem.

Corollary 2.1 [5, Theorem 2.1] Let $n>1, \quad r \geq 0, s \geq 0$ be fixed non-negative integers and let $R$ be a ring with unity 1 in which for every $y \in R$ there exist integers $l=l(y) \geq 0, \quad j=j(y) \geq 0, \quad m=m(y) \geq 0 \quad$ such that $x^{r}\left[x^{n}, y\right] x^{s}=y^{i}\left[x, y^{m}\right] y^{j}$ for all $x \in R$. Further, if at least one of $r, s$ is zero and $R$ satisfies the property $Q(n)$, then $R$ is commutative.

The existence of unity 1 in the hypothesis of Theorem 2.1 can be justified by the following example.

Example 2.1 Let $D_{k}$ be the ring of all $k \times k$ matrices over a division ring $D$ and $A_{k}=\left\{\left(a_{i j} \in D_{k} / a_{i j}=0(i \geq j)\right\}\right.$. Then $A_{3}$ is a non-commutative ring of index 3 which satisfies the identity $\left[x^{n}, y\right]=\left[x, y^{n}\right]$ for all $x, y \in A_{3}$ and $n \geq 2$.

The following example strengthens the existence of the property $Q(n)$ in the hypothesis of the Theorem 2.1 .

Example 2.2 Let $R=\left\{\left(\begin{array}{lll}a & b & c \\ 0 & a & d \\ 0 & 0 & a\end{array}\right) \quad / a, b, c, d \in G F(2)\right\}$.

Then $R$ is a non-commutative ring with unity 1 satisfying the identity $\left[x^{n}, y\right]=\left[x, y^{n}\right]$ for all $x, y \in R$ and $n=2$.

\section{Commutativity of Rings Through Streb's Classification}

In an attempt to generalize a well known theorem due to Bell [7], Quadri and Khan [31] proved; a ring $R$ with unity 1 is commutative if it satisfies a polynomial identity $\left[x y-y^{m} x^{n}, x\right]=0$ where $m \geq 1$ and $n \geq 1$ are positive integers. Further Bell et al [6] established that the above result remains true if the value of the exponent $m$ appearing in the given identity is no longer fixed rather depends on the ring element $y$. Recently, Nishinaka [27] improved this result as follows: a ring $R$ with unity 1 is commutative if it satisfies the condition $\left[x^{m} y-f(y) x^{n}, x\right]=0$ for some $f(t) \in t^{2} Z[t]$, where $m$ and $n$ are fixed non-negative integers. The objective of the present paper is to further extend the study in this direction and investigate the commutativity of a ring $R$ satisfying the following properties:

$(\mathrm{CH})$ For each $x, y \in R$ there exists a polynomial $f(X) \in Z[X]$ such that $\left[x-x^{2} f(x), y-y^{2} f(y)\right]=0$

$\left(P_{1}\right)$ For each $x, y \in R$ there exists an integer $m \geq 0$ and polynomials $f(X) \in X^{2} Z[X]$ and $g(X), h(X) \in X Z[X]$ such that $\left\{1-x^{m} y g\left(x^{m} y\right)\right\}$ $\left[x^{m} y-x^{m} y f\left(x^{m} y\right), x\right]\left\{1-x^{m} y h\left(x^{m} y\right)\right\}=0$.

$\left(P_{1}^{*}\right)$ For each $x, y \in R$ there exists an integer $m=m(x, y) \geq 0$ and polynomials $f(X) \in X^{2} Z[X]$ and $g(X), h(X) \in X Z[X]$ such that $\left\{1-x^{m} y g\left(x^{m} y\right)\right\}$ $\left[x^{m} y-x^{m} y f\left(x^{m} y\right), x\right]\left\{1-x^{m} y h\left(x^{m} y\right)\right\}=0$.

$\left(P_{2}\right)$ For each $x, y \in R$ there exists an integer $m \geq 0$ and polynomials $f(X) \in X^{2} Z[X]$ and $g(X), h(X) \in X Z[X]$ such that $\left\{1-x^{m} y g\left(x^{m} y\right)\right\}\left[y x^{m}-x^{m} y f\left(x^{m} y\right), x\right]\left\{1-x^{m} y h\left(x^{m} y\right)\right\}=0$.

$\left(P_{2}^{*}\right)$ For each $x, y \in R$ there exists an integer $m=m(x, y) \geq 0$ and polynomials $f(X) \in X^{2} Z[X]$ and $g(X), h(X) \in X Z[X]$ such that

$$
\left\{1-x^{m} y g\left(x^{m} y\right)\right\}\left[y x^{m}-x^{m} y f\left(x^{m} y\right), x\right]\left\{1-x^{m} y h\left(x^{m} y\right)\right\}=0
$$

The discussion starts with the following theorem:

Theorem 3.1 Let $R$ be a ring with unity 1 satisfying either of the property $\left(P_{1}\right)$ or $\left(P_{2}\right)$, then $R$ is commutative (and conversely).

The following rings should be taken into consideration in order to develop the proof of the above theorem:

$$
\begin{array}{ll}
\text { (i) } & \left(\begin{array}{cc}
G F(p) & G F(p) \\
0 & G F(p)
\end{array}\right) \\
\text { (i) })_{l} & \left(\begin{array}{cc}
G F(p) & G F(p) \\
0 & 0
\end{array}\right) \\
\text { (i) } r & \left(\begin{array}{cc}
G F(p) & 0 \\
G F(p) & 0
\end{array}\right) \\
\text { (ii) } & M_{\sigma}(K)=\left\{\left(\begin{array}{cc}
\alpha & \beta \\
0 & \sigma(\alpha)
\end{array}\right) / \alpha, \beta \in K\right\}, \text { where } K \text { is a }
\end{array}
$$

finite field with a non-trivial automorphism.

(iii) A non-commutative division ring.

(iv) $\mathrm{S}=<1>+T, T$ a non-commutative radical subring of $S$.

(v) $\mathrm{S}=<1>+T, T$ a non-commutative radical subring of $S$ such that $T[T, T]=[T, T] T=0$.

In the year 1989, Streb [33] had given the classification of non-commutative rings which has been used effectively as a tool to obtain a number of commutativity theorems (cf.[22], [23], [24] further references can be found). From the proof of [34, corollary 1], it can be easily observe that if $R$ is a non- 
commutative ring with unity 1 , then there exists a factor subring of $R$ which is of the form (i), (ii), (iii), (iv) or (v). This observation gives the following result which plays the key role in our subsequent study (cf., [24, Lemma 1].

Lemma 3.1 Let $P$ be a ring property which is inherited by factor subrings. If there no rings of the form $(i),(i i),(i i i)$, (iv) or ( $v$ ) satisfy the property $P$, then every ring with unity 1 satisfying the property $P$ is commutative.

The following result has been proved in [22, Corollary 1].

Lemma 3.2 Suppose that a ring $R$ with unity 1 satisfies the condition $(\mathrm{CH})$ and if $R$ is a non-commutative ring, then it is always possible to find out a factor subring of $R$ which is of the form $(i)$ or $(i i)$.

The following result is due to Herstein [13].

Lemma 3.3 Let $R$ be a ring in which for every $x, y \in R$ there exists polynomial $f(X) \in Z[X]$ such that $\left[x-x^{2} f(x), y\right]=0, R$ then is commutative.

The proof of the following lemma is essential for developing the proof of the main theorem.

Lemma 3.4 If $R$ is a division ring satisfying either of the property $\left(P_{1}\right)$ or $\left(P_{2}\right)$, then $R$ is commutative.

Proof. Suppose $R$ satisfies the property $\left(P_{1}\right)$. Let $u$ be a unit in $R$, then for every $y \in R$ there exist polynomials $f(X) \in X^{2} Z[X]$ and $g(X), h(X) \in X Z[X]$ such that

$0=\left\{1-\left(x^{-m} x^{m} y\right) g\left(x^{-m} x^{m} y\right)\right\}\left[x^{-m} x^{m} y-x^{-m} x^{m} y f\left(x^{-m} x^{m} y\right), x\right.$

$$
\begin{aligned}
& \left\{1-\left(x^{-m} x^{m} y\right) h\left(x^{-m} x^{m} y\right)\right\} \\
& \quad=\{1-y g(y)\}[y-y f(y), x]\{1-y h(y)\} .
\end{aligned}
$$

This implies that $1-y g(y)=0$ or $1-y h(y)=0$ or $[y-y f(y), x]=0$. In all possible cases, $R$ is commutative by application of Lemma 3.3.

Proof of the Theorem. Suppose $R$ satisfies the property $\left(P_{1}\right)$. In view of Lemma 3.1, it is sufficient enough to show that $R$ do not belongs to the family of considered rings. First consider, $R$ is a ring of the form $\left(\begin{array}{cc}G F(p) & G F(p) \\ 0 & G F(p)\end{array}\right)$, $\left(\begin{array}{cc}G F(p) & G F(p) \\ 0 & 0\end{array}\right)$ or $\left(\begin{array}{cc}G F(p) & 0 \\ G F(p) & 0\end{array}\right)$. Then in $(G F(p))_{2}$, where $p$ is a prime then it can be observe that for each $f(X) \in X^{2} Z[X]$ and $g(X), h(X) \in X Z[X]$

$$
\begin{aligned}
& \left\{1-e_{11}^{m}\left(e_{12}+e_{22}\right) g\left(e_{11}^{m}\left(e_{12}+e_{22}\right)\right)\right\} \\
& {\left[e_{11}^{m}\left(e_{12}+e_{22}\right)-e_{11}^{m}\left(e_{12}+e_{22}\right) f\left(e_{11}^{m}\left(e_{12}+e_{22}\right), e_{11}\right]\right.} \\
& \left\{1-e_{11}^{m}\left(e_{12}+e_{22}\right) h\left(e_{11}^{m}\left(e_{12}+e_{22}\right)\right)\right\} \\
& =-e_{12} \neq 0,
\end{aligned}
$$

a contradiction. Hence, there is no ring of the form

$$
\left(\begin{array}{cc}
G F(p) & G F(p) \\
0 & G F(p)
\end{array}\right),\left(\begin{array}{cc}
G F(p) & G F(p) \\
0 & 0
\end{array}\right) \text { or }\left(\begin{array}{cc}
G F(p) & 0 \\
G F(p) & 0
\end{array}\right)
$$

which is satisfying the property $\left(P_{1}\right)$. If we consider the ring $R=M_{\sigma}(K)=\left\{\left(\begin{array}{cc}\alpha & \beta \\ 0 & \sigma(\alpha)\end{array}\right) / \alpha, \beta \in K\right\}$, where $K$ is taken as a finite field with a non-trivial automorphism. Now, choosing

$$
x=\left(\begin{array}{cc}
\alpha & 0 \\
0 & \sigma(\alpha)
\end{array}\right), y=\left(\begin{array}{ll}
0 & 1 \\
0 & 0
\end{array}\right)
$$

Then for each $f(X) \in X^{2} Z[X] \quad$ and $g(X), h(X) \in X Z[X]$ we see that

$$
\begin{gathered}
\left\{1-x^{m} y g\left(x^{m} y\right)\right\}\left[x^{m} y-x^{m} y f\left(x^{m} y\right), x\right]\left\{1-x^{m} y h\left(x^{m} y\right)\right\} \\
=\alpha^{m}(\sigma(\alpha)-\alpha) e_{12} \neq 0
\end{gathered}
$$

again a contradiction, therefore $R \neq M_{\sigma}(K)$. Further, suppose that $R$ is a non-commutative division ring, then by the application of Lemma 3.4, $R$ is commutative, a contradiction. Assume that $R=\mathrm{S}=<1>+T, T$ a noncommutative radical subring of $S$, then a careful scrutiny of the proof of Lemma 3.4 shows that for a unit $u \in R$ and arbitrary $y \in R$, there exist polynomials $q(X) \in X^{2} Z[X]$ and $g(X), h(X) \in X Z[X]$ such that either $1-y g(y)=0$ or $1-y h(y)=0$ or $[y-y f(y), x]=0$. Let $a, b \in T$, then $1+a$ is a unit and there exist polynomials $q(X) \in X^{2} Z[X]$ and $g(X), h(X) \in X Z[X]$ such that either $1-b g(b)=0$ or $1-b h(b)=0$ or $[1+a, b-b(q)]=0$. Hence by Lemma 3.3, $T$ is commutative, a contradiction. Now, suppose that $R=\mathrm{S}=<1>+T, T$ a non-commutative radical subring of $S$ such that $T[T, T]=[T, T] T=0$. Then for each $a, b \in T$ there exist polynomials $f(X) \in X^{2} Z[X] \quad$ and $g(X), h(X) \in X Z[X]$ such that

$$
\begin{gathered}
0=\left\{1-(1+a)^{m} \operatorname{bg}\left((1+a)^{m} b\right)\right\} \\
{\left[(1+a)^{m} b-(1+a)^{m} b f(1+a)^{m} b, 1+a\right]} \\
\left\{1-(1+a)^{m} b h\left((1+a)^{m} b\right)\right\} \\
=\left\{1-(1+a)^{m} b g\left((1+a)^{m} b\right)\right\}\left[(1+a)^{m} b, 1+a\right] \\
\left\{1-(1+a)^{m} b h\left((1+a)^{m} b\right)\right\} \\
=\left\{1-(1+a)^{m} b g\left((1+a)^{m} b\right)\right\}[b, 1+a] \\
\left\{1-(1+a)^{m} b h\left((1+a)^{m} b\right)\right\} \\
=[b, a]
\end{gathered}
$$

This is again a contradiction, hence $R \neq \mathrm{S}=<1>+T, \quad T$ a 
non-commutative radical subring of $S$ such that $T[T, T]=[T, T] T=0$. Now, Let $R$ satisfies the property $\left(P_{2}\right)$. If $R$ is a ring of the form $\left(\begin{array}{cc}G F(p) & G F(p) \\ 0 & G F(p)\end{array}\right)$, $\left(\begin{array}{cc}G F(p) & G F(p) \\ 0 & 0\end{array}\right)$ or $\left(\begin{array}{ll}G F(p) & 0 \\ G F(p) & 0\end{array}\right)$, then there exist polynomials $f(X) \in X^{2} Z[X]$ and $g(X), h(X) \in X Z[X]$ such that

$$
\begin{aligned}
& \left\{1-e_{22}^{m} e_{12} g\left(e_{22}^{m} e_{12}\right)\right\}\left[e_{12} e_{22}^{m}-e_{22}^{m} e_{12} f\left(e_{22}^{m} e_{12}\right), e_{22}\right] \\
& \left\{1-e_{22}^{m} e_{12} h\left(e_{22}^{m} e_{12}\right)\right\} \\
& \quad=e_{12} \neq 0,
\end{aligned}
$$

a contradiction. Using similar arguments as used above, It can be easily shown that there does not exists any ring among the remaining four rings as discussed above which is satisfying the property $\left(P_{2}\right)$ and in view of Lemma 3.1, we get the required result.

Corollary 3.1 Let $m \geq 0$ be a fixed positive integer and let $R$ be a ring with unity 1 . If for each $x, y \in R$ there exists a polynomial $f(X) \in X^{2} Z[X] \quad$ such that $\left[x^{m} y-x^{m} y f\left(x^{m} y\right), x\right]=0$, then $R$ is commutative.

Remark 3.1 If the integral exponent $m$ in the properties $\left(P_{1}\right)$ and $\left(P_{2}\right)$ are allowed to vary with the pair of ring elements $x, y \in R$, i.e. $R$ satisfies either of the property $\left(P_{1}^{*}\right)$ or $\left(P_{2}^{*}\right)$, then a careful scrutiny of the proof of Theorem 3.1 shows that $R$ has no factor subring of the type (i) or (ii). Thus in addition, if $R$ satisfies the property $(\mathrm{CH})$, then in view of Lemma 3.2, we get the following:

Theorem 3.2 Let $R$ be a ring with unity 1 satisfying either of the property $\left(P_{1}^{*}\right)$ or $\left(P_{2}^{*}\right)$. Moreover, if $R$ satisfies the property $(\mathrm{CH})$, then $R$ is commutative (and conversely).

Example 3.1 The non-commutative ring of $3 \times 3$ strictly upper triangular matrices over a ring satisfies the property $\left[x^{m} y-x^{m} y f\left(x^{m} y\right), x\right]=0$ and hence rules out the possible generalization of the above theorem for arbitrary rings.

\section{Commutativity of $s$-Unital Rings}

A ring $R$ is called left (respectively right) $s$ - unital ring if $x \in R x$ (respectively $x \in x R$ ) for all $x \in R$. Further $R$ is called $s$ - unital ring if $R$ is both left as well as right $S$ - unital i.e. $x \in R x \cap x R$ for all $x \in R$. Following [16], if $R$ is $S$ - unital ring (respectively left or right $S$ - unital ring) then for any subset $F$ of $R$ there exists an element $e \in R$ such that $e x=x e=x$ respectively $x e=e x=x$ for all $x \in F$. Such an element $e$ is called pseudo identity (respectively left or right identity) of $F$ in $R$. There are numerous examples in the existing literature (cf. [24, Remark 2]) which shows these classes of rings are generalization of class of rings with unity. Recently, many results for rings with unity 1, particularly a number of commutativity theorems have been extended to one sided $s$ - unital ring .

Although Example 2.1 strengthens the existence of unity 1 in the hypothesis of Theorem 2.1, the same theorem may be extended to one sided $s$ - unital rings.

Theorem 4.1 Let $n>1, k>0, m \geq 0, r \geq 0, s \geq 0$ be fixed non-negative integers and let $R$ be a left (respectively right) $S$ - unital ring in which for every $y \in R$ there exist polynomials $\quad p(X), q(X) \in Z[X]$ such that $x^{r}\left[x^{n}, y\right]=p(y)\left[x, y^{m}\right]^{k} q(y)$ (resp. $\left[x^{n}, y\right] x^{s}=p(y)\left[x, y^{m}\right]^{k} q(y)$ for all $x \in R$. If $R$ satisfies the property $Q(n)$, then $R$ is commutative.

Proof. Let $R$ be a left (resp. right) $S$ - unital ring satisfying $x^{r}\left[x^{n}, y\right]=p(y)\left[x, y^{m}\right]^{k} q(y)$ (resp. $\left[x^{n}, y\right] x^{s}=p(y)\left[x, y^{m}\right]^{k} q(y) \quad$ for some $p(X), q(X) \in Z[X]$. Choose an element $e$ in $R$ such that $e x=x, e y=y(\operatorname{resp} . x e=x, y e=y)$. Now replace $x$ by $e$ in the given property, we get

$$
\begin{gathered}
e^{r}\left[\mathrm{e}^{n}, y\right]=p(y)\left[\mathrm{e}, y^{m}\right]^{k} q(y) \text { (resp. } \\
{\left[e^{n}, y\right] e^{s}=p(y)\left[\mathrm{e}, y^{m}\right]^{k} q(y)}
\end{gathered}
$$

for some $p(X), q(X) \in Z[X]$ and fixed integers $n>1, \quad k>0, m \geq 0, r \geq 0, s \geq 0$. This yields that $\mathrm{y}=y \mathrm{e}^{n} \in y R$ (resp. $y=\mathrm{e}^{n} y \in R y$ ). Thus $R$ is right (resp. left) $s$ - unital ring and hence $s$ - unital. Now in view of proposition [15], we can assume that $R$ has unity 1 and hence commutativity of $R$ follows by Theorem 2.1

Corollary 4.1 [5, Theorem 2.3] Let $n>1, r \geq 0, s \geq 0$ be fixed non-negative integers and let $R$ be a left (resp. right) $s$ - unital ring in which for $\forall y \in R$ there exists integers $t=t(y) \geq 0, j=j(y) \geq 0, m=m(y) \geq 0 \quad$ such that $x^{r}\left[x^{n}, y\right]=y^{t}\left[x, y^{m}\right] y^{j}$ (resp. $\left.\left[x^{n}, y\right] x^{s}=y^{t}\left[x, y^{m}\right] y^{j}\right)$ for all $x \in R$. If $R$ satisfies the property $Q(n)$, then $R$ is commutative.

The following example shows that a left (respectively right) $s-$ unital ring with the property $\left[x^{n}, y\right] x^{s}=y^{t}\left[x, y^{m}\right] y^{j} \quad\left(\right.$ resp. $\left.\quad x^{r}\left[x^{n}, y\right]=y^{t}\left[x, y^{m}\right] y^{j}\right)$ need not be commutative.

Example 4.1

Let $R_{1}=\left\{\left(\begin{array}{ll}0 & 0 \\ 0 & 0\end{array}\right), \quad\left(\begin{array}{ll}1 & 0 \\ 1 & 0\end{array}\right), \quad\left(\begin{array}{ll}0 & 1 \\ 0 & 1\end{array}\right), \quad\left(\begin{array}{ll}1 & 1 \\ 1 & 1\end{array}\right)\right\} \quad$ be $\quad$ a subring of $2 \times 2$ matrices over $G(F(2))$. Then $R_{1}$ is a noncommutative left $s$ - unital ring satisfying the property $\left[x^{n}, y\right] x^{s}=y^{t}\left[x, y^{m}\right] y^{j}$ for any fixed positive integers $n>1, k>0 \quad r \geq 0, \quad s \geq 0, t \geq 0, j \geq 0$. Indeed $R_{1}$ has the property $Q(n)$ for odd $n$. 


\section{Similarly}

$R_{2}=\left\{\left(\begin{array}{ll}0 & 0 \\ 0 & 0\end{array}\right), \quad\left(\begin{array}{ll}1 & 1 \\ 0 & 0\end{array}\right), \quad\left(\begin{array}{ll}0 & 0 \\ 1 & 1\end{array}\right), \quad\left(\begin{array}{ll}1 & 1 \\ 1 & 1\end{array}\right)\right\} \quad$ be $\quad \mathrm{a}$ subring of $2 \times 2$ matrices over $G(F(2))$. Then $R_{2}$ is a noncommutative right $s$ - unital ring satisfying the property $x^{r}\left[x^{n}, y\right]=y^{t}\left[x, y^{m}\right] y^{j}$ for any fixed positive integers $n>1, k>0 \quad r \geq 0, \quad s \geq 0, t \geq 0, j \geq 0$. Indeed $R_{2}$ has the property $Q(n)$ for odd $n$.

Before moving ahead to establish commutativity of $s$ unital rings satisfying some related properties as considered in Theorem 3.1, the following lemma is due to Komatsu et al. [25] is pertinent in order to make our paper self-contained.

Lemma 4.1 If $R$ is left $s$ - unital not right $s$ - unital, then $R$ has a factor subring of the form $\left(\begin{array}{cc}G F(p) & G F(p) \\ 0 & 0\end{array}\right)$ Theorem 4.2 Let $R$ be a left (respectively right) $s$ - unital ring in which for every $x, y \in R$ there exists polynomial $f(X) \in X^{2} Z[X]$ such that $\left[x^{m} y-x^{m} y f\left(x^{m} y\right), x\right]=0$ resp. $\left[y x^{m}-x^{m} y f\left(x^{m} y\right), x\right]=0$ where $m \geq 0$ is a fixed positive integer. Then $R$ is commutative (and conversely).

Proof. If $R$ is a left (respectively right) $s$-unital ring satisfying the identity $\left[x^{m} y-x^{m} y f\left(x^{m} y\right), x\right]=0$ respectively $\left[y x^{m}-x^{m} y f\left(x^{m} y\right), x\right]=0$, then a careful scrutiny of the proof of Theorem 3.1 shows that no ring of the form $\left(\begin{array}{cc}G F(p) & G F(p) \\ 0 & 0\end{array}\right)$ respectively $\left(\begin{array}{cc}G F(p) & 0 \\ G F(p) & 0\end{array}\right)$ satisfy the property $\quad\left[x^{m} y-x^{m} y f\left(x^{m} y\right), x\right]=0 \quad$ resp. $\left[y x^{m}-x^{m} y f\left(x^{m} y\right), x\right]=0$ Hence, by Lemma $4.1, R$ is right (respectively left) $s$-unital. Thus, in both the cases $R$ is $s-$ unital and in view of Proposition 1 of [15], it can be assumed that $R$ has unity 1 and commutativity of $R$ follows by Theorem 3.1.

Using the similar arguments as used to get Theorem 3.2, the following can be easily proved:

Theorem 4.3 Let $R$ be a left (respectively right) $s$-unital ring in which for every $x, y \in R$ there exists integer $m=m(x, y) \geq 0$ and a polynomial $f(X) \in X^{2} Z[X]$ such that $\quad\left[x^{m} y-x^{m} y f\left(x^{m} y\right), x\right]=0 \quad$ resp. $\left[y x^{m}-x^{m} y f\left(x^{m} y\right), x\right]=0$. Moreover, $R$ also satisfies the property $(\mathrm{CH})$. Then $R$ is commutative (and conversely).

Conclusion. Bell proved the commutativity of a ring $R$ with unity 1 satisfying the polynomial identity $\left[x^{n}, y\right]=\left[x, y^{n}\right]$ if the additive group $(R,+)$ is $\mathrm{n}$-torsion free. This result of Bell possess a natural question that weather it is feasible to extend this result for some wider polynomial identities or not. This paper gives an affirmative answer of this question and hence establishes commutativity of a ring with unity 1 satisfying some wider polynomial identities and further assures the commutativity of one sided $s$-unital rings also. The same results may also be extended to another class of rings called near rings as well as derivations of rings.

\section{References}

[1] Abujabal, H. A. S.: on commutativity of left $s$ - unital rings, Acta Sci. Math. 56(1992). 51-62.

[2] Abujabal, H. A. S., Obaid, M. A.: Some commutativity theorems for right $s$ - unital rings, Math. Japonica 37 (1999), 591-600.

[3] Abujabal, H. A. S. and Ashraf M.: On commutativity of rings involving certain polynomial Constraints, Algebra Colloq. 5(1998), 111-116.

[4] Ashraf M.: A commutativity theorem for associative rings, Arch. Math (Brno) 31 (1995), 201-204.

[5] Ashraf M.: Commutativity of rings with certain constraints, Proc. R. Ir. Acad. 96 A, No. 2 (1996), 177-183.

[6] Bell, H. E., Quadri, M. A. and Ashraf, M.: Commutativity of rings with some commutator constraints, Rad. Mat. 5(1989), 223-230.

[7] Bell H. E.:A commutativity condition for rings, Canad. J. Math. 28(1979), 986-991.

[8] Bell H. E.: On power maps and ring commutativity, Canad. Mathematics Bulletin 21(1978), 399-404.

[9] Bell H. E.: The identity $(x y)^{n}=x^{n} y^{n}$, does it buy commutativity, Mathematics Mag. 55(1982), 165-170.

[10] Bell H. E.: Commutativity of rings with constraints on commutators, Resultate Math. 8 (1985), 123-131.

[11] Harmanci, A.: Two elementary commutativity theorems for rings, Acta. Math. Sci. Hungar 29(1977), 23-29.

[12] Herstein, I. N.: Two remarks on commutativity of rings, Canadian Journal Math. 9(1957), 583-586.

[13] Herstein, I. N.: Power maps in rings, Michigan J. 8(1961), 583-586.

[14] Hirano, Y., Kobayashi, Y. and Tominaga, H: Commutativity theorems for certain rings, Math. J. Okayama Univ. 22(1980), 65-72.

[15] Hirano, Y., Kobayashi, Y. and Tominaga, H: Some polynomial identities and Commutativity of $s$ - unital rings, Math. J. Okayama Univ. 24(1982), 7-13.

[16] Hirano, Y., Hongan, H. and Tominaga, H: Commutativity theorems for certain rings, Math. J. Okayama Univ. 22(1980), 65-72.

[17] Jacobson, N.: Structure theory of algebraic algebras of bounded degree, Ann. of Math. 46(1945), 695-707.

[18] Jacobson, N.: Structure of rings, Amer. Math. Soc. Colloq. Publ. 37(1964).

[19] Kezlan, T. P.: A not on commutativity of semi-prime PI rings, Math Japonica 26 (1982), 267-268.

[20] Komatsu H.: A commutativity theorem for rings, Math. Journal. Okayam Univ. 26(1984), 135-139.

[21] Komatsu H.: A commutativity theorem for rings-II, Osaka Journal of Math. 22 (1985), 811-814. 
[22] Komatsu H. and Tominaga, H.: Chacron's conditions and commutativity theorems, Math. Journal Okayam Univ. 31(1989), 101-120.

[23] Komatsu H. and Tominaga, H.: Some commutativity theorems for $s$ - unital rings, Resultate Math. 15(1989), 335-342.

[24] Komatsu H. and Tominaga, H.: Some commutativity conditions for rings with unity, Resultate Math. 19(1991), 8388 .

[25] Komatsu H., Nishinaka, T. and Tominaga, H.: On commutativity of rings, Radovi Mat. 6(1990), 303-311.

[26] Khan, M. S.;Rings admitting certain decomposition theorems. Journal of Advances in Mathemativs, Vol. 13, No. 01, 12017.

[27] Nishinaka, T.: A commutativity of rings, Rad. Mat. 6(1990), 357-359.

[28] Psomopoulos, E.: A commutativity theorem for rings involving a subset of ring, Glasnik Mat. 8(1983), 231-236.

[29] Psomopoulos, E.: Commutativity theorem for rings and groups, Internat. Journal of Math. and Math. Sci. 7 No. 3 (1984), 513-517.
[30] Psomopoulos, E., Tominaga, H. and Yaqub, A.,: Some commutativity theorems for $\mathrm{n}$ - torsion free rings, Math. Journal of Okayama Univ. 23(1981), 37-39.

[31] Quadri, M. A. and Khan, M. A.: A commutativity theorem for associative ring, Math. Japonica 29(1984), 371-373.

[32] Raza, M. A., Khan, M. S. and Rehamn, Nadeem-ur; Derivation in prime and semiprime rings with Banach algebras, Italian Journal of Pure and applied Mathematics, Vol. 40, 2018 (To Appear).

[33] Streb's, W.: Zur struktur nichtkommutativer ringe, Math. J. Okayama Univ. 31(1989), 135-140.

[34] Tominaga, H. and Yaqub, A.: Commutativity theorems for rings with constraints involving a commutative subset, Resultate Math. 11(1987), 186-192.

[35] Tominaga, H.: Some commutativity theorems for s - unital rings satisfying certain constraint, Resultate Math. 6(1983), 217-219. 